\title{
Phytohormone signaling pathway for eliciting leaf phenolic synthesis in honeysuckle (Lonicera japonica Thunb.) under coastal saline environment
}

\author{
Kun Yan ${ }^{\mathrm{a}, 1, *}$, Lanxing Bian ${ }^{\mathrm{a}, \mathrm{b}, 1}$, Wenjun He ${ }^{\mathrm{a}, 1}$, Guangxuan Han ${ }^{\mathrm{a}}$, Zishan Zhang ${ }^{\mathrm{c}}$, Zheng Sun ${ }^{\mathrm{d}}$, \\ Likun Liang $^{\mathrm{b}}$, Hongxin Jia ${ }^{\mathrm{b}}$, Guangmei Wang ${ }^{\mathrm{a}}{ }^{\mathrm{*}}$ \\ ${ }^{a}$ CAS Key Laboratory of Coastal Environmental Processes and Ecological Remediation, Yantai Institute of Coastal Zone Research (YIC), Chinese Academy of Sciences \\ (CAS), Shandong Key Laboratory of Coastal Environmental Processes, YICCAS, Yantai, Shandong 264003, PR China \\ ${ }^{\mathrm{b}}$ College of Life Sciences, Yantai University, Yantai 264005, PR China \\ ' State Key Laboratory of Crop Biology, Shandong Key Laboratory of Crop Biology, College of Life Sciences, Shandong Agricultural University, Tai'an 271018, PR China \\ d Dongjiakou Port Customs, Qingdao, PR China
}

\section{A R T I C L E I N F O}

\section{Keywords:}

Chinese medicinal crop

Coastal saline land

Field trial

Gene transcription

Phenolic synthesis

Signal transduction

\begin{abstract}
A B S T R A C T
To date, it remains unclear how saline stress induces leaf phenolic synthesis in honeysuckle (Lonicera japonica Thunb.), a traditional Chinese medicinal crop. This study was to investigate hormone signaling for salt-induced leaf phenolic synthesis in honeysuckle from molecular, physiological and ecological levels by pot experiments and field trials. As a major ingredient of soil salinity in coastal land, $\mathrm{NaCl}$ was used in salt treatment for simulating coastal saline environment. $\mathrm{NaCl}$ stress significantly increased leaf abscisic acid (ABA) and salicylic acid (SA) concentrations, potentiated leaf phenolic synthesis by elevating phenylalanine ammonia-lyase (PAL) activity and PALs transcription, and enhanced leaf phenolic accumulation. Under $\mathrm{NaCl}$ stress, tungstate and paclobutrazol pretreatments inhibited leaf ABA and SA accumulation, respectively, and lowered PAL activity, PALs transcription, and phenolic accumulation. Thus, ABA and SA participated in transducing salt-induced leaf phenolic synthesis. Paclobutrazol pretreatment hardly influenced leaf ABA concentration in salt-treated plants, but tungstate pretreatment abated salt-induced leaf SA accumulation, indicating that ABA was an upstream signal of SA. Under $\mathrm{NaCl}$ stress, SA application restored leaf SA level with no effect on ABA level but incompletely restored PAL activity and phenolic accumulation in tungstate-pretreated plants, confirming the downstream role of SA and implying the existence of other downstream signals. Leaf ABA and SA concentrations were higher in plants in coastal saline plots than non-saline plots, and the roles of ABA and SA in mediating salt-induced leaf phenolic synthesis passed ecological test, according to a significant positive correlation of leaf ABA and SA levels with phenolic concentration. In conclusion, ABA acted as an upstream signal of SA to elicit leaf phenolic synthesis in honeysuckle under coastal saline environment.
\end{abstract}

\section{Introduction}

Under saline stress, plants are confronted with osmotic pressure, ionic toxicity and secondary oxidative stress, and take use of adaptive mechanisms to maintain ion, water, and reactive oxygen species (ROS) homostasis (Munns and Tester, 2008; Hossain and Dietz, 2016; Van Zelm et al., 2020). Phenolic synthesis is an salt adaptive response for helping control ROS level in plant tissues, but less attention has been paid to its induction mechanism compared with stomatal closure, osmolyte synthesis, and $\mathrm{Na}^{+}$exclusion (Cheynier et al., 2013; Sah et al.,

\section{6; Zhu, 2016).}

Plant phenolics comprising flavonoids, phenolic acids, and tannins are a major kind of secondary metabolites. Recent studies attached importance to bioactive phenolic ingredients in plants for producing functional foods, cosmetics and medicines (Shahidi and Ambigaipalan, 2015; Van Hung, 2016; Dra et al., 2019; Gunes et al., 2019; Hazrati et al., 2019; Al Juhaimi et al., 2020), and also investigated phenolic synthesis without concerning environmental disturbance (Liang et al., 2013; Park et al., 2015; Jia et al., 2017; Xing et al., 2018; Wang et al., 2019; Waseem et al., 2019). Phenolics are capable to scavenge ROS, and

\footnotetext{
* Corresponding authors at: Yantai Institute of Coastal Zone Research, Chinese Academy of Sciences, Chunhui road 17, Yantai 264003, China.

E-mail addresses: kyan@yic.ac.cn, yankunacademic@163.com (K. Yan), gmwang@yic.ac.cn (G. Wang).

1 The authors contributed equally to this work
} 
phenolic accumulation can strengthen antioxidant defense under environmental stresses (Grace and Logan, 2000; Cheynier et al., 2013). To date, phenolic accumulation has been widely reported in plants exposed to salt stress, but phenolic synthesis is rarely emphasized, let alone its signaling pathway. As a traditional viewpoint, phenolics play a secondary role in antioxidant protection in contrast to the primary antioxidant system involving superoxide dismutase, ascorbate peroxidase, ascorbate, etc. (Ferreres et al., 2011; Fini et al., 2011; Agati et al., 2013). Thus, present studies mainly focus on signal transduction for the major salt tolerance mechanisms such as $\mathrm{Na}^{+}$transportation, osmotic regulation and primary antioxidants rather than phenolic synthesis (Jayakannan et al., 2013; Osakabe et al., 2014; Dinneny, 2015; Hossain and Dietz, 2016; Per et al., 2017; Yan et al., 2018). However, phenolic synthesis becomes very important from the view of plant nutrition, and notably, saline stress can improve the value of medicinal crops by elevating accumulation of bioactive phenolic compounds (Rouphael et al., 2018; Yan et al., 2016a). So far, synergic variation of plant hormones with phenolic concentration has been reported in some plants under salt stress (Zhu et al., 2016; El-Esawi et al., 2017; Ibrahim et al., 2019; Khaleghnezhad et al., 2019; Mousavi and Shabani, 2019; Perin et al., 2019), but it still lacks sufficient evidence whether these hormones participate in mediating salt-induced phenolic synthesis. In a word, the signaling pathway for salt-induced plant phenolic synthesis remains far from clear.

Plant hormones are a series of endogenous signal molecules and regulate many physiological metabolisms. In the numerous plant hormones, abscisic acid (ABA) is defined as a stress hormone because of its crucial role in eliciting adaptive behaviors. For instance, salt-induced leaf stomatal closure and root $\mathrm{Na}^{+}$exclusion depend on ABA signaling pathway (Osakabe et al., 2014; Sah et al., 2016; Yan et al., 2018). Likewise, salicylic acid (SA) also can induce salt adaptive responses such as osmolyte synthesis and prevention of $\mathrm{K}^{+}$loss (Jayakannan et al., 2013; Per et al., 2017). At present, the promotional effects of ABA and SA on plant phenolic accumulation have been reported (Yang et al., 2012; Liang et al., 2013; Alonso et al., 2016; Shakirova et al., 2016; El-Esawi et al., 2017; Golkar et al., 2019; Khaleghnezhad et al., 2019; Mousavi and Shabani, 2019), whereas the inconsistent results in some studies imply that the signal transduction for phenolic synthesis is species specific (Li et al., 2010; Buran et al., 2012; Felicijan et al., 2016). Recently, ABA and SA were definitely demonstrated as signal molecules to elicit phenolic synthesis, respectively, in the leaves of Aristotelia chilensis upon drought stress and in the leaves of Camellia sinensis with elevated $\mathrm{CO}_{2}$ exposure (Gonzalez-Villagra et al., 2019; Li et al., 2019). However, it remains not explicit whether ABA and SA mediate salt-induced plant phenolic synthesis, and moreover, the cross-talk between SA and ABA is largely unknown.

As a traditional Chinese medicinal crop, honeysuckle (Lonicera japonica Thunb.) is rich in bioactive phenolics such as chlorogenic acid, and has been widely used for producing various Chinese medicines including some important antiviral medicines. In our recent studies, salt tolerant honeysuckle cultivar was screened for planting in coastal saline land, and the field trials proved its capacity to desalinize coastal soil (Yan et al., 2015, 2016b). Most importantly, soil salinity improved its medicinal quality by elevating phenolic and chlorogenic acid accumulation (Yan et al., 2016a). Thus, it is of great advantage to utilize honeysuckle for exploiting coastal abandoned saline land. It should be stressed that leaf phenolic accumulation resulted from elevated phenolic synthesis in honeysuckle under coastal saline environment, however, hormone signals for eliciting leaf phenolic synthesis were still unknown (Yan et al., 2017). To date, almost all studies on signal transduction are conducted by using pot experiments, and lack ecological proof and practical value. This study was intended to explore the roles of ABA and SA in salt-induced leaf phenolic synthesis in honeysuckle from molecular and physiological levels by pot experiments and verify their function from the ecological level by field trials in coastal saline land. We hypothesized that ABA cooperated with SA to induce leaf phenolic synthesis in honeysuckle under saline stress. Our study can provide insight into phenolic synthetic mechanism in plants, and may present a valuable guidance for agricultural practice in coastal saline land.

\section{Materials and methods}

\subsection{Room pot experiments}

Bear root honeysuckle plants were placed in plastic pots containing Hoagland nutrient solution and grown ( $\mathrm{pH}$ 5.7) in climatic chambers (Qiushi, China). The nutrient solution was continuously aerated and refreshed once every two days. Photosynthetic active radiation intensity was controlled at $200 \mu \mathrm{mol} \mathrm{m}^{-2} \mathrm{~s}^{-1}$ for $12 \mathrm{~h}$ (07:00 to 19:00) every day, and day/night temperature and humidity were set at $25 / 18^{\circ} \mathrm{C}$ and $65 \%$ in the chambers. Forty days later, uniform plants were separated to seven groups. Tungstate sodium and paclobutrazol are widely used as specific inhibitors, respectively, for ABA and SA biosynthesis (Jiang and Zhang, 2002; Ren and Dai, 2012; Guajardo et al., 2016; Li et al., 2019). As a major ingredient of soil salinity in coastal land, $\mathrm{NaCl}$ is commonly used to conduct salt treatment for simulating coastal saline environment (Yan et al., 2016a, b; Yan et al., 2017). In the first group, control plants were pretreated by deionized water without subsequent exposure to $\mathrm{NaCl}$ stress. In the second group, plants were pretreated by tungstate sodium without subsequent exposure to $\mathrm{NaCl}$ stress. In the third group, plants were pretreated by paclobutrazol without subsequent exposure to $\mathrm{NaCl}$ stress. In the fourth group, plants were exposed to $200 \mathrm{mM} \mathrm{NaCl}$ for six days after pretreatment with water. In the fifth group, plants were exposed to $200 \mathrm{mM} \mathrm{NaCl}$ for six days after pretreatment with tungstate sodium. In the sixth group, plants were exposed to $200 \mathrm{mM} \mathrm{NaCl}$ for six days after pretreatment with paclobutrazol. In the seventh group, plants were pretreated by tungstate sodium, applied with exogenous SA, and then exposed to $200 \mathrm{mM} \mathrm{NaCl}$ for six days. One day before $\mathrm{NaCl}$ treatment, the attached leaves were immersed in tungstate sodium $(1 \mathrm{mM})$ and paclobutrazol $(0.1 \mathrm{mM})$ solutions for $5 \mathrm{~min}$ to inhibit ABA and SA synthesis, and tungstate-pretreated leaves were immersed in SA $(50 \mu \mathrm{M})$ solution for $5 \mathrm{~min}$ for SA application. Tungstate sodium, paclobutrazol and SA were bought from sigma. Five replicate plants in each group were randomly selected to sample newly expanded leaves for parameter measurements.

\subsection{Field trials}

Field plots were established in Dongying Halophyte Arboretum in Shandong province, China $\left(37^{\circ} 24^{\prime} \mathrm{N}, 118^{\circ} 39^{\prime} \mathrm{E}\right)$. Climate conditions, soil background and plot design have been described in our previous studies (Yan et al., 2016b, 2017). Generally, there were four replicate non-saline and saline plots, and the electronic conductance of tilth soil was $486 \mu \mathrm{s}$ $\mathrm{cm}^{-1}$ and $910 \mu \mathrm{sm}^{-1}$, respectively. Three plants were randomly selected in each plot to collect newly expanded leaves in growth seasons.

\subsection{Measurements of $A B A$ and $S A$ concentrations}

ABA and SA concentrations were simultaneously analyzed according to Liu et al. (2012). ABA and SA were extracted from fresh leaf tissues, and liquid chromatography mass spectrometry was used to determine their concentrations. The extraction procedure, liquid chromatography process and analyses of mass spectrum have been illuminated in our recent study (Yan et al., 2018). In this study, ABA and SA were quantified by monitoring 263/153 and 137/93 transition based on MS/MS method.

\subsection{The in situ visualization of phenolics}

Small fresh leaf pieces were sampled and embedded in $3 \%$ agarose. Transverse sections $(40 \mathrm{~mm}$ ) were prepared by using a vibrating blade microtome (Leica VT1200, Germany), immersed in absolute methanol 

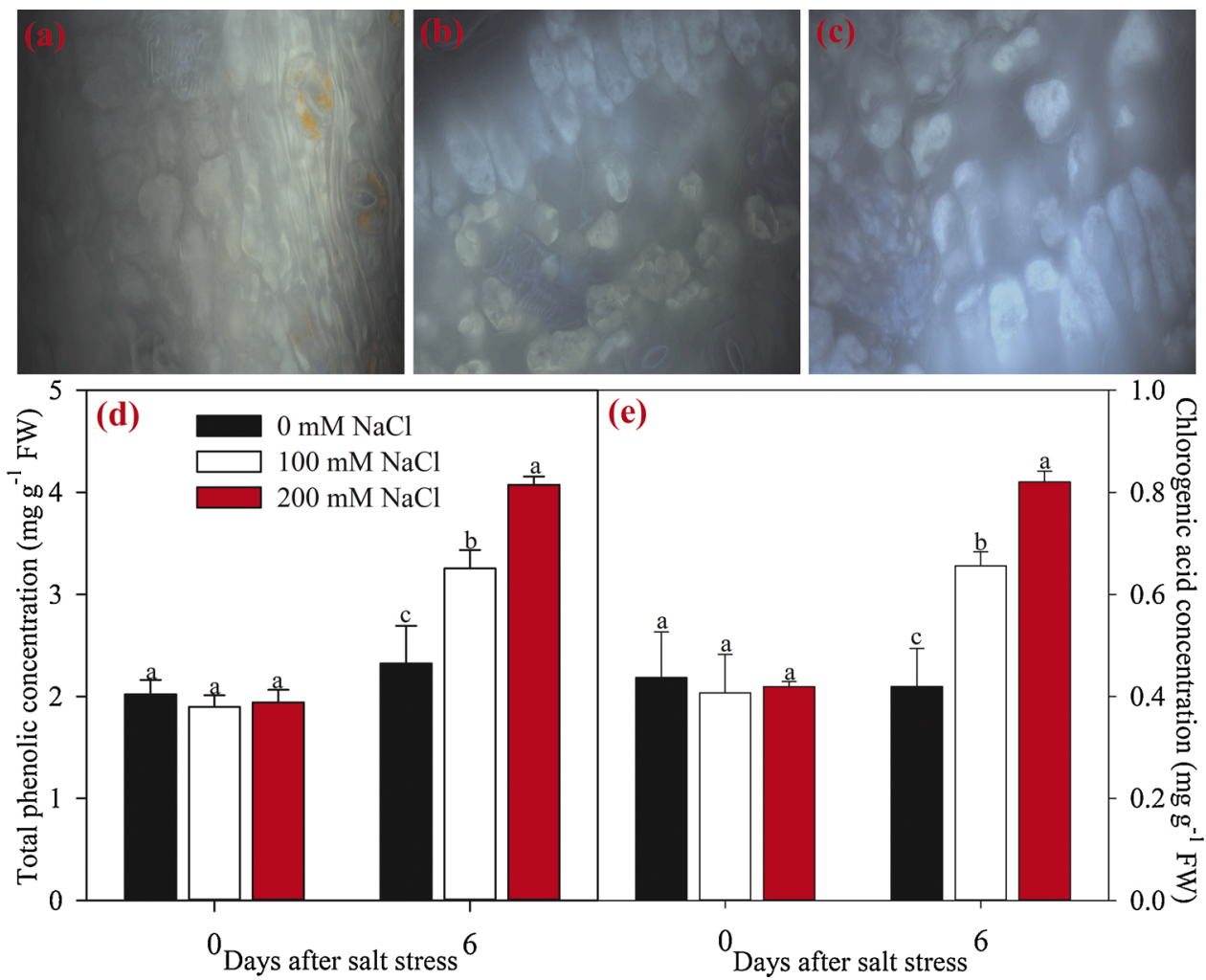

Fig. 1. Changes in leaf total phenolic (d) and chlorogenic acid (e) concentrations in honeysuckle after salt stress and in situ visualization of leaf phenolics with enlargement of 100 times in control plants without $\mathrm{NaCl}$ stress (a) and plants exposed to $100 \mathrm{mM} \mathrm{(b)} \mathrm{and} 200 \mathrm{mM}$ (c) NaCl stress for 6 days. FW indicates fresh weight. Data are the mean of five replicates $( \pm \mathrm{SD})$ in the figure. Significant differences at $P<0.05$ are represented by different letters on error bars.

for $1 \mathrm{~min}$ for clearing the chlorophyll, and then mounted in $90 \%$ glycerol solution. The leaf sections were viewed under a fluorescence microscope (Olympus BX53, Japan) with UV light (365 nm excitation). After excitation by UV light, phenolics, particularly the caffeic acid esters, can specifically emit blue fluorescence at $400-430 \mathrm{~nm}$ (Hutzler et al., 1998).

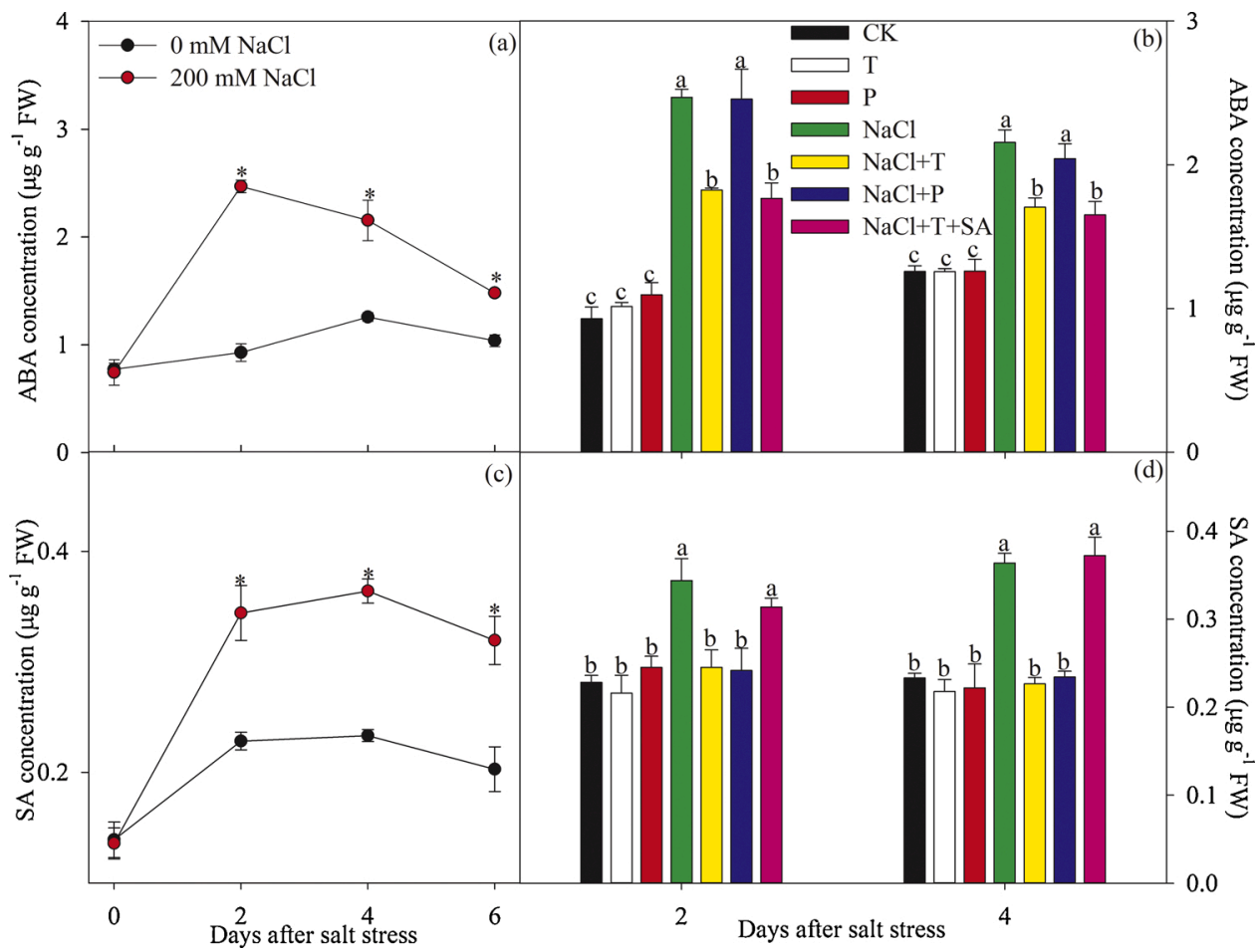

Fig. 2. Variation of leaf $A B A$ and $S A$ concentrations during salt stress $(\mathrm{a}, \mathrm{c})$ and leaf $\mathrm{ABA}$ and SA concentrations in tungstate-pretreated and paclobutrazol-pretreated honeysuckle under salt stress (b, d). FW indicates fresh weight. Data are the mean of five replicates $( \pm$ SD) in the figure. Significant differences at $P$ $<0.05$ are represented by different letters on error bars and asterisks. CP indicates control plants without exposure to $\mathrm{NaCl}$ stress; $\mathrm{T}$ indicates tungstate-pretreated plants without exposure to $\mathrm{NaCl}$ stress; $\mathrm{P}$ indicates paclobutrazol-pretreated plants without exposure to $\mathrm{NaCl}$ stress; $\mathrm{N}$ indicates plants exposed to $200 \mathrm{mM} \mathrm{NaCl}$ stress; NT indicates plants exposed to $200 \mathrm{mM} \mathrm{NaCl}$ stress after tungstate pretreatment; NP indicates plants exposed to $200 \mathrm{mM} \mathrm{NaCl}$ stress after paclobutrazol pretreatment; NTS indicates plants exposed to 200 $\mathrm{mM} \mathrm{NaCl}$ stress after tungstate pretreatment and SA application. The symbols, CP, T, P, N, NT, NP and NTS are also used in the following figures. 


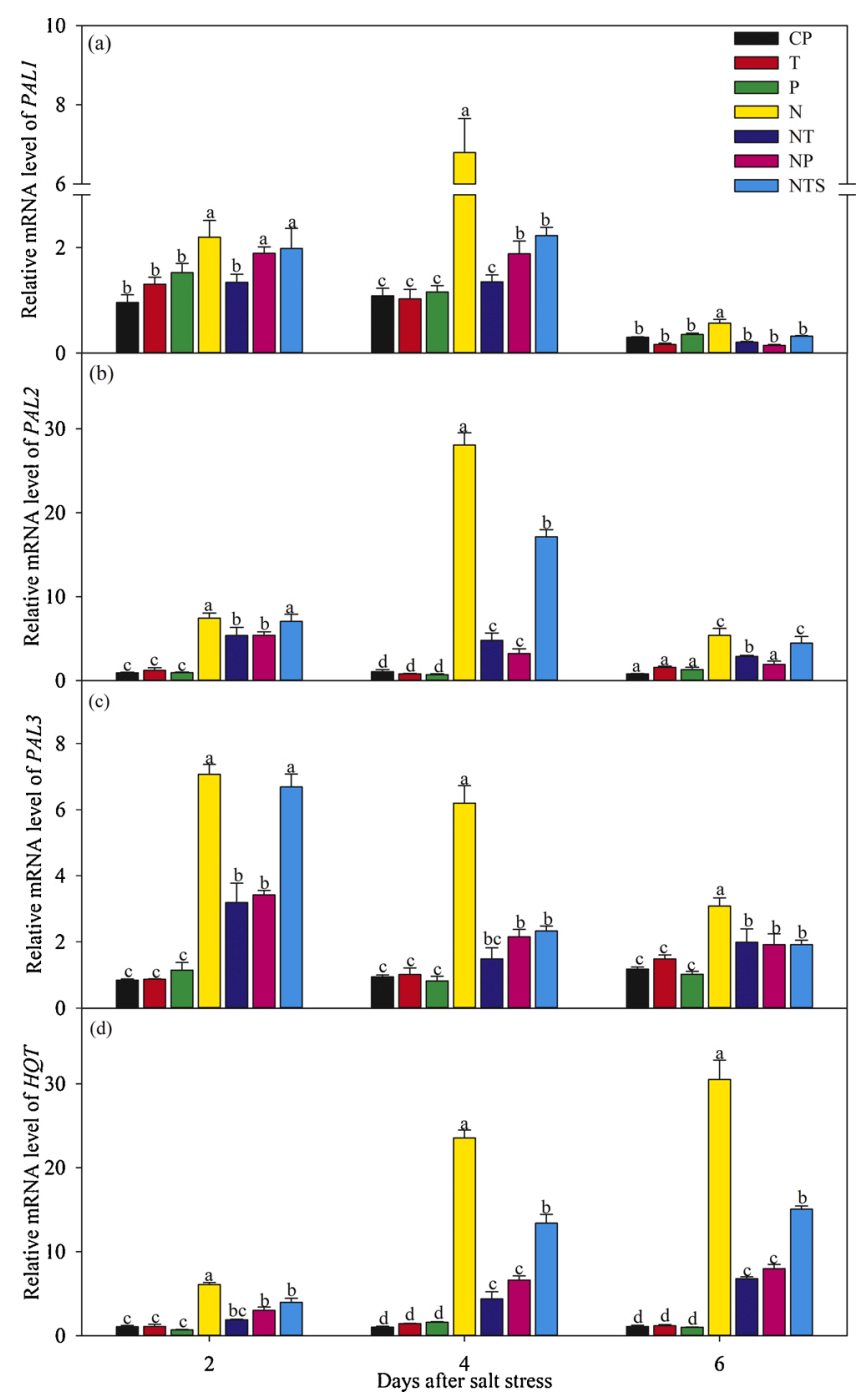

Fig. 3. Gene transcription of leaf phenylalanine ammonia-lyase (PAL, a-c) and hydroxycinnamoyl-CoA quinate: hydroxycinnamoyl transferase (HQT, d) in tungstate-pretreated and paclobutrazol-pretreated honeysuckle under salt stress. The genes, PAL1, PAL2 and PAL3 belong to PAL gene family. Data are the mean of five replicates $( \pm S D)$ in the figure. Significant differences at $P<0.05$ are represented by different letters on error bars.

\subsection{Measurements of total phenolic and chlorogenic acid concentrations and phenylalanine ammonia-lyase (PAL) activity}

Fresh leaves were homogenized in $60 \%$ methanol after grinding with liquid nitrogen. The mixture was centrifuged to collect supernatant for measuring total phenolic and chlorogenic acid concentrations (Yan et al., 2017). Fresh leaves were homogenized in borate buffer ( $\mathrm{pH} 8.8$ ) and centrifuged after grinding with liquid nitrogen, and the supernatant was used for measuring PAL activity (Yan et al., 2017).

\subsection{Real time quantitative PCR analysis}

The experiments of real time quantitative PCR were carried out for analyzing transcription of PAL and hydroxycinnamoyl-CoA quinate: hydroxycinnamoyl transferase (HQT) by using the same internal reference gene, primers and equipment in our recent study (Yan et al., 2016a).

\subsection{Statistical analysis}

The presented data are the mean of five replicate plants in pot experiments or samples collected from four replicate plots. All kinds of data were compared through one-way ANOVA by using SPSS 16.0 (SPSS Inc., Chicago, IL, USA) through LSD test, and difference was considered significant at $P<0.05$. SPSS 16.0 was also used to perform regression analysis.

\section{Result}

\subsection{Effects of salt stress on leaf total phenolic and chlorogenic acid} concentrations

Leaf total phenolic and chlorogenic acid concentrations were markedly elevated after 6 days of $100 \mathrm{mM} \mathrm{NaCl}$ stress, and the increase was up to $75.4 \%$ and $95.5 \%$ after 6 days of $200 \mathrm{mM} \mathrm{NaCl}$ stress (Fig. 1d and e). As shown in Fig. 1a-c, the specific blue fluorescence gradually increased with the aggravation of $\mathrm{NaCl}$ stress, verifying salt-induced leaf phenolic accumulation.

\subsection{Effects of $A B A$ and $S A$ synthetic inhibitors on their concentrations under salt stress}

Leaf ABA and SA concentrations were remarkably elevated after 2 days of $\mathrm{NaCl}$ stress, and they were maintained at a significantly higher level in salt-treated plants than control plants in following days (Fig. 2a and c). Tungstate and paclobutrazol did not obviously influence leaf $\mathrm{ABA}$ and SA concentrations, however, salt-induced increase in leaf ABA and SA concentrations were significantly dampened in tungstatepretreated and paclobutrazol-pretreated plants (Fig. 2b and d). Tungstate pretreatment also abated salt-induced increase in leaf SA concentration, whereas salt-induced increase in leaf ABA concentration was not affected by paclobutrazol pretreatment (Fig. 2b and d). Notably, SA application restored leaf SA concentration but had no effect on leaf ABA concentration in tungstate-pretreated plants under salt stress (Fig. 2b and d).

\subsection{Effects of $A B A$ and $S A$ synthetic inhibitors on genes transcription under salt stress}

Tungstate and paclobutrazol did not obviously influence the transcription of PAL1, PAL2, PAL3 and HQT, but their transcription was remarkably elevated by $\mathrm{NaCl}$ stress (Fig. 3). Tungstate and paclobutrazol pretreatments depressed salt-induced elevation in transcription of PAL1, $P A L 2, P A L 3$ and HQT, and the depression was partly mitigated by SA application in tungstate-pretreated plants (Fig. 3).

\subsection{Effects of $A B A$ and SA synthetic inhibitors on PAL activity and phenolic concentration under salt stress}

Tungstate and paclobutrazol did not obviously influence PAL activity, total phenolic and chlorogenic acid concentrations in the leaves (Fig. 4). NaCl stress significantly increased leaf PAL activity by $61.4 \%$, $41.2 \%$ and $110.4 \%$, respectively, at day 2,4 and 6 , and consistently, leaf total phenolic and chlorogenic acid concentrations were remarkably increased by $75.4 \%$ and $95.5 \%$ after 6 days of $\mathrm{NaCl}$ stress (Fig. 4). Saltinduced elevation in leaf PAL activity, total phenolic and chlorogenic acid concentrations was significantly depressed in tungstate-pretreated and paclobutrazol-pretreated plants (Fig. 4). The depression in tungstate-pretreated plants was greater and could be partly alleviated by SA application (Fig. 4). 

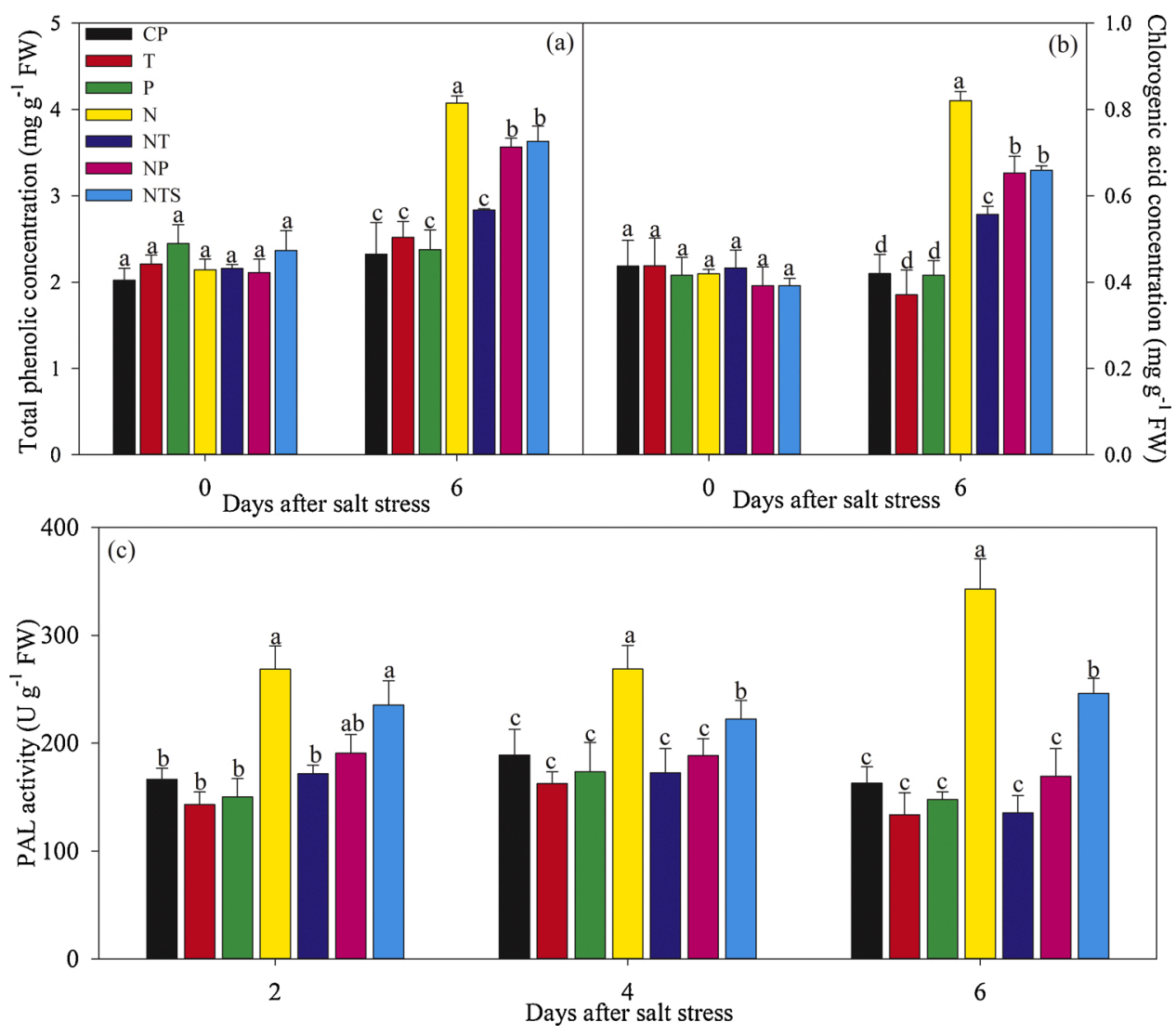

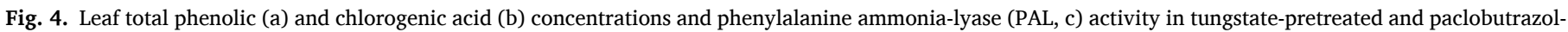

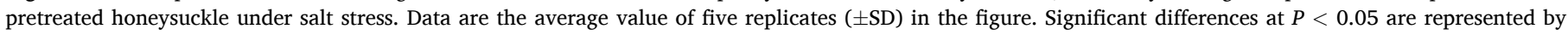
different letters on error bars.

\subsection{Correlation of leaf $A B A$ and $S A$ concentrations with total phenolic concentration}

In growth seasons of 2014 and 2015, leaf ABA and SA concentrations were higher in plants in saline plots than non-saline plots, and insignificant difference arose in some individual months (Fig. 5a and b). The regression analysis suggested a significant positive correlation of leaf $\mathrm{ABA}$ and SA concentrations with total phenolic concentration (Fig. 5c and d).

\section{Discussion}

Consistent with our previous study (Yan et al., 2017), salt stress enhanced leaf total phenolic and chlorogenic acid concentrations in honeysuckle and was beneficial to its medicinal quality (Fig. 1d and e). In this study, salt-induced leaf phenolic accumulation was confirmed by in situ visualization through fluorescence microscopy (Fig. 1a-c), and leaf phenolic synthesis was also elevated by salt stress according to increased PAL activity and transcription of PALs and HQT (Figs. 3 and 4c). As the crucial hormone signals, ABA and SA are commonly accumulated in plants for defensive purposes upon environmental stresses, and phenolic synthesis and accumulation can be enhanced by exogenous applying ABA and SA in some plants (Alonso et al., 2016; El-Esawi et al., 2017; Golkar et al., 2019; Khaleghnezhad et al., 2019; Mousavi and Shabani, 2019). Therefore, higher leaf ABA and SA concentrations appeared in parallel with elevated phenolic synthesis in salt-treated honeysuckle, implying that ABA and SA potentially participated in eliciting phenolic synthesis under salt stress.

However, it remains not definite whether ABA and SA can serve as signal molecules to induce phenolic synthesis in plants under salt stress. As expected, salt-induced leaf ABA accumulation was effectively depressed in honeysuckle by tungstate (Fig. 2b). The declined leaf ABA concentration inhibited phenolic synthesis by abating salt-induced increase in PAL activity (Fig. 4c), which also could be verified from molecular level by lower transcription of PALs and HQT in tungstatepretreated plants than non-pretreated plants upon salt stress (Fig. 3). Correspondingly, salt-induced leaf total phenolic and chlorogenic acid accumulation was dampened in tungstate-pretreated plants (Fig. 4a and b). Thus, salt-induced leaf phenolic synthesis in honeysuckle depended on ABA signaling pathway. As with $\mathrm{ABA}$, SA was also proved to be engaged in triggering leaf phenolic synthesis under salt stress from physiological and molecular levels, because the declined SA accumulation reduced leaf phenolic and chlorogenic acid concentrations and inhibited PAL activity and transcription of PALs and HQT under salt stress (Figs. 3 and 4). Therefore, ABA and SA mediated salt-induced leaf phenolic synthesis in honeysuckle. In fact, the induction of physiological adaptive responses relies on signaling networks rather than a single hormone (Golldack et al., 2014), and particularly, Li et al. (2019) also reported that SA cooperated with NO to induce leaf flavonoid synthesis in tea plant under elevated $\mathrm{CO}_{2}$. In this study, tungstate pretreatment reduced not only $\mathrm{ABA}$ accumulation but also $\mathrm{SA}$ accumulation in the leaves under salt stress (Fig. $2 b$ and d), suggesting that ABA-dependent SA production was responsible for phenolic synthesis, and unchanged leaf ABA level in paclobutrazol-pretreated plants upon salt stress also corroborated that $\mathrm{ABA}$ was an upstream signal (Fig. 2b). Overall, salt-induced leaf phenolic synthesis was mediated by ABA and SA signaling in honeysuckle, and ABA acted as an upstream signal of SA. It was noteworthy that tungstate pretreatment led to greater reduction of total phenolic and chlorogenic acid concentrations in salt-treated plants than paclobutrazol pretreatment, and so was in PAL activity (Fig. 4). This result was in accordance with the role of ABA as upstream signal, and it also indicated that apart from SA, there might be other unknown 

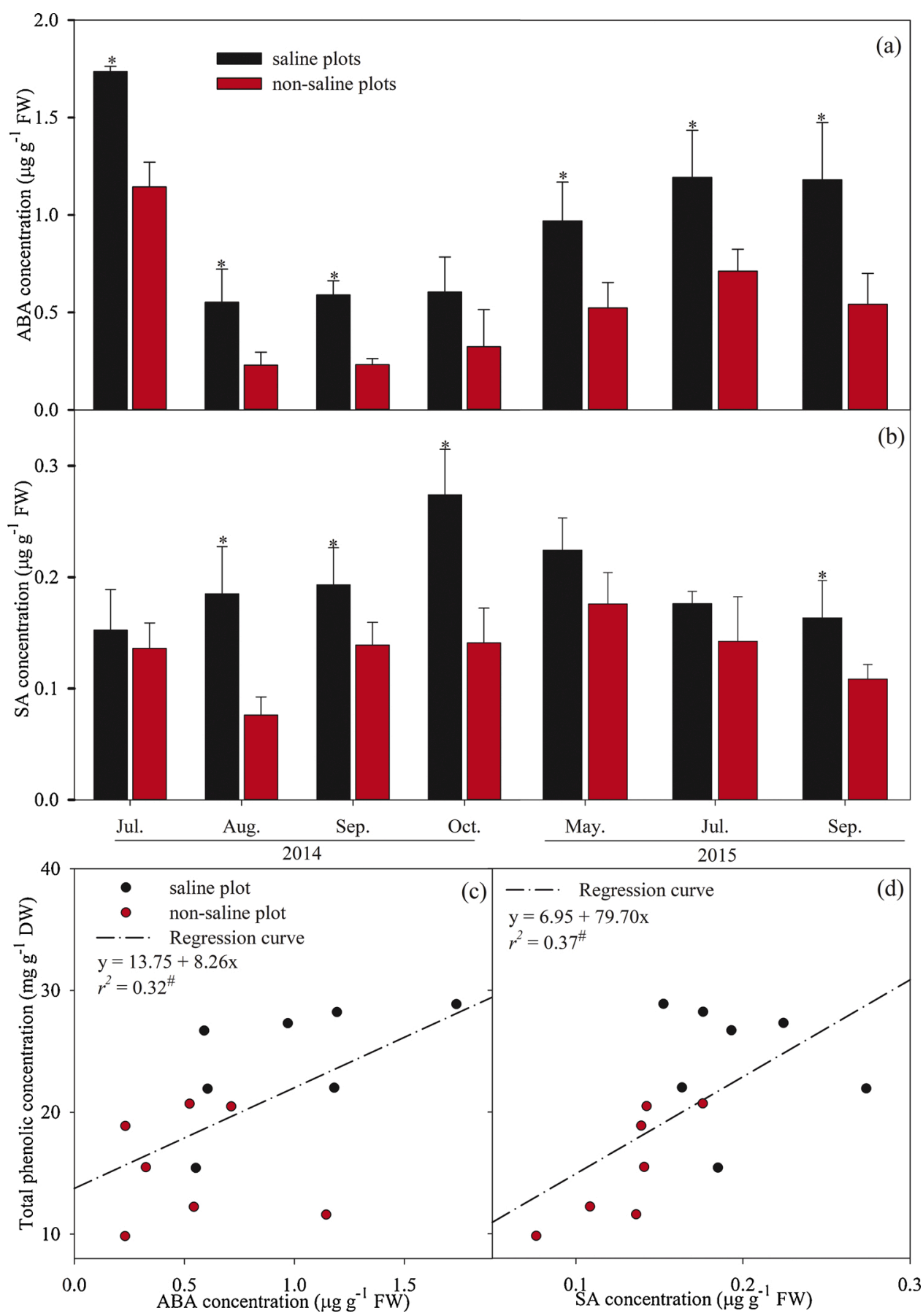

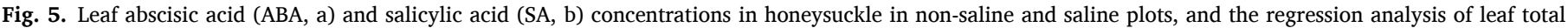

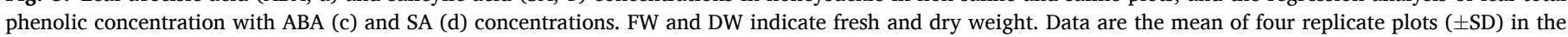
figure. Significant difference at $P<0.05$ is represented by asterisks on error bars, and significant correlation at $P<0.05$ was indicated by \#.

downstream signals to mediate ABA-dependent phenolic synthesis under salt stress. Moreover, this inference was underpinned by the fact that SA application incompletely restored leaf phenolic synthesis and concentration in tungstate-pretreated plants under salt stress (Fig. 4). Besides ABA and SA, other signal molecules such as brassinosteroid, ethylene, and methyl jasmonate have been reported to elicit plant phenolic synthesis (Yang et al., 2012; Liang et al., 2013; Coban and Gokturk Baydar, 2016), and they are proper candidate signals for deeply exploring salt-induced leaf phenolic synthesis in honeysuckle in future study. ABA and SA were preferentially selected in this study, because of their low price and convenience for application in agricultural practice.

To date, the results about signal transduction for physiological metabolisms are rarely examined by field trial and lack an ecological proof. In agreement with pot experiments, leaf ABA and SA concentrations in honeysuckle were also enhanced by soil salinity in the field trials (Fig. 5a and b). Hormones production might be interrupted by environmental factors such as precipitation, light and temperature. Consequently, leaf $\mathrm{ABA}$ and SA levels exhibited seasonal changes, and possibly due to the dilution effects of environmental factors, the differences of leaf ABA and SA concentrations between plants in saline and non-saline plots became insignificant in some individual months (Fig. 5a and b). It should be emphasized that a significant positive correlation of leaf ABA and SA levels with total phenolic concentration was demonstrated in honeysuckle under coastal saline environment (Fig. $5 c$ and d), and this 
finding conformed to the signaling transduction mechanism revealed by hydroponic experiments. In other words, the viewpoint that salt-induced leaf phenolic synthesis was mediated by ABA and SA in honeysuckle passed ecological test and had important practical value.

In agreement with our hypothesis, ABA acted as an upstream signal of SA to elicit leaf phenolic synthesis in honeysuckle under salt stress, according to the analysis at molecular and physiological levels. This finding was verified from ecological angle under coastal saline environment, providing a critical guidance for agricultural practice in coastal abandoned saline land.

\section{CRediT authorship contribution statement}

Kun Yan: Methodology. Lanxing Bian: Methodology. Wenjun He: Methodology. Guangxuan Han: Methodology. Zishan Zhang: Methodology. Zheng Sun: Methodology. Likun Liang: Methodology. Hongxin Jia: Methodology. Guangmei Wang: Methodology.

\section{Declaration of Competing Interest}

The authors report no declarations of interest.

\section{Acknowledgments}

This study was financed by Shandong Provincial Natural Science Foundation, China (ZR2017QC005), National Natural Science Foundation of China (41201292), Yantai Science and Technology Innovation Development Plan (2020MSGY065), National Key Research \& Development Program in China (2019YFD1002702) and Key Deployment Project of Chinese Academy of Sciences (KFZD-SW-113). We express our sincere thanks to Prof. Huiyuan Gao for critically reading this manuscript.

\section{References}

Agati, G., Brunetti, C., Di Ferdinando, M., Ferrini, F., Pollastri, S., Tattini, M., 2013 Functional roles of flavonoids in photoprotection: new evidence, lessons from the past. Plant Physiol. Biochem. 72, 35-45.

Al Juhaimi, F., Ghafoor, K., Uslu, N., Ahmed, I.A.M., Babiker, E.E., Ozcan, M.M. Fadimu, G.J., 2020. The effect of harvest times on bioactive properties and fatty acid compositions of prickly pear (Opuntia ficus-barbarica A. Berger) fruits. Food Chem. 303, 125387.

Alonso, R., Berli, F.J., Fontana, A., Piccoli, P., Bottini, R., 2016. Malbec grape (Vitis vinifera L.) responses to the environment: berry phenolics as influenced by solar UVB, water deficit and sprayed abscisic acid. Plant Physiol. Biochem. 109, 84-90.

Buran, T.J., Sandhu, A.K., Azeredo, A.M., Bent, A.H., Williamson, J.G., Gu, L., 2012. Effects of exogenous abscisic acid on fruit quality, antioxidant capacities, and phytochemical contents of southern high bush blueberries. Food Chem. 132, 1375-1381.

Cheynier, V., Comte, G., Davies, K.M., Lattanzio, V., Martens, S., 2013. Plant phenolics: recent advances on their biosynthesis, genetics, and ecophysiology. Plant Physiol. Biochem. 72, 1-20.

Coban, O., Gokturk Baydar, N., 2016. Brassinosteroid effects on some physical and biochemical properties and secondary metabolite accumulation in peppermint (Mentha piperita L.) under salt stress. Ind. Crop. Prod. 86, 251-258.

Dinneny, J.R., 2015. Traversing organizational scales in plant salt-stress responses. Curr. Opin. Plant Biol. 23, 70-75.

Dra, L.A., Rodrigues, M.J., Neng, N.D., Nogueira, J.M.F., Elamine, Y., Aghraz, A., Markouk, M., Larhsini, M., Custodio, L., 2019. Exploring Caralluma europaea (Guss.) NEBr. As a potential source of bioactive molecules: in vitro antioxidant and antidiabetic properties, and phenolic profile of crude extracts and fractions. Ind. Crop. Prod. 139, 111527.

El-Esawi, M.A., Elansary, H.O., El-Shanhorey, N.A., Abdel-Hamid, A.M.E., Ali, H.M., Elshikh, M.S., 2017. Salicylic acid-regulated antioxidant mechanisms and gene expression enhance rosemary performance under saline conditions. Front. Physiol. 8, 716.

Felicijan, M., Kristl, J., Krajnc, A.U., 2016. Pre-treatment with salicylic acid induces phenolic responses of Norway spruce (Picea abies) bark to bark beetle (Ips typographus) attack. Trees 30, 2117-2129.

Ferreres, F., Figueiredo, R., Bettencourt, S., Carqueijeiro, I., Oliveira, J., GilIzquierdo, A., Pereira, D.M., Valentao, P., Andrade, P.B., Duarte, P., Ros Barcelo, A., Sottomayor, M., 2011. Identification of phenolic compounds in isolated vacuoles of the medicinal plant Catharanthus roseus and their interaction with vacuolar class III peroxidase: an $\mathrm{H}_{2} \mathrm{O}_{2}$ affair? J. Exp. Bot. 62, 2841-2854.
Fini, A., Brunetti, C., Di Ferdinando, M., Ferrini, F., Tattini, M., 2011. Stress-induced flavonoid biosynthesis and the antioxidant machinery of plants. Plant Signal. Behav. 6, 709-711.

Golkar, P., Taghizadeh, M., Yousefian, Z., 2019. The effects of chitosan and salicylic acid on elicitation of secondary metabolites and antioxidant activity of safflower under in vitro salinity stress. Plant Cell Tissue Organ Cult. 137, 575-585.

Golldack, D., Li, C., Mohan, H., Probst, N., 2014. Tolerance to drought and salt stress in plants: unraveling the signaling networks. Front. Plant Sci. 5, 151.

Gonzalez-Villagra, J., Cohen, J.D., Reyes-Diaz, M.M., 2019. Abscisic acid is involved in phenolic compounds biosynthesis, mainly anthocyanins, in leaves of Aristotelia chilensis plants (Mol.) subjected to drought stress. Physiol. Plant. 165, 855-866.

Grace, S.C., Logan, B.A., 2000. Energy dissipation and radical scavenging by the plant phenylpropanoid pathway. Phil. Trans. R. Soc. Lond. 355, 1499-1510.

Guajardo, E., Correa, J.A., Contreras-Porcia, L., 2016. Role of abscisic acid (ABA) in activating antioxidant tolerance responses to desiccation stress in intertidal seaweed species. Planta 243, 767-781.

Gunes, A., Kordali, S., Turan, M., Bozhuyuk, A.U., 2019. Determination of antioxidant enzyme activity and phenolic contents of some species of the Asteraceae family from medicanal plants. Ind. Crop. Prod. 137, 208-213.

Hazrati, S., Ebadi, M.T., Mollaei, S., Khurizadeh, S., 2019. Evaluation of volatile and phenolic compounds, and antioxidant activity of different parts of Ferulago angulata (schlecht.) Boiss. Ind. Crop. Prod. 140, 7.

Hossain, M.S., Dietz, K.J., 2016. Tuning of redox regulatory mechanisms, reactive oxygen species and redox homeostasis under salinity stress. Front. Plant Sci. 7, 548.

Hutzler, P., Fischbach, R., Heller, W., Jungblut, T.P., Reuber, S., Schmitz, R., Veit, M., Weissenbock, G., Schnitzler, J.P., 1998. Tissue localization of phenolic compounds in plants by confocal laser scanning microscopy. J. Exp. Bot. 49, 953-965.

Ibrahim, W., Zhu, Y.M., Chen, Y., Qiu, C.W., Zhu, S., Wu, F., 2019. Genotypic differences in leaf secondary metabolism, plant hormones and yield under alone and combined stress of drought and salinity in cotton genotypes. Physiol. Plant. 165, 343-355.

Jayakannan, M., Bose, J., Babourina, O., Rengel, Z., Shabala, S., 2013. Salicylic acid improves salinity tolerance in Arabidopsis by restoring membrane potential and preventing salt-induced $\mathrm{K}^{+}$loss via a GORK channel. J. Exp. Bot. 64, 2255-2268.

Jia, Y., Bai, Z., Pei, T., Ding, K., Liang, Z., Gong, Y., 2017. The protein kinase SmSnRK2.6 positively regulates phenolic acid biosynthesis in Salvia miltiorrhiza by interacting with SmAREB1. Front. Plant Sci. 8, 1384.

Jiang, M.Y., Zhang, J.H., 2002. Water stress-induced abscisic acid accumulation triggers the increased generation of reactive oxygen species and up-regulates the activities of antioxidant enzymes in maize leaves. J. Exp. Bot. 53, 2401-2410.

Khaleghnezhad, V., Yousefi, A.R., Tavakoli, A., Farajmand, B., 2019. Interactive effects of abscisic acid and temperature on rosmarinic acid, total phenolic compounds, anthocyanin, carotenoid and flavonoid content of dragonhead (Dracocephalum moldavica L.). Sci. Hortic. 250, 302-309.

Li, Z., Zhao, X., Sandhu, A.K., Gu, L., 2010. Effects of exogenous abscisic acid on yield, antioxidant capacities, and phytochemical contents of greenhouse grown lettuces. J. Agric. Food Chem. 58, 6503-6509.

Li, X., Zhang, L., Ahammed, G.J., Li, Y.T., Wei, J.P., Yan, P., Zhang, L.P., Han, X., Han, W. Y., 2019. Salicylic acid acts upstream of nitric oxide in elevated carbon dioxideinduced flavonoid biosynthesis in tea plant (Camellia sinensis L.). Environ. Exp. Bot. $161,367-374$.

Liang, Z., Ma, Y., Xu, T., Cui, B., Liu, Y., Guo, Z., Yang, D., 2013. Effects of abscisic acid, gibberellin, ethylene and their interactions on production of phenolic acids in Salvia miltiorrhiza bunge hairy roots. PLoS One 8, e72806.

Liu, H., Li, X., Xiao, J., Wang, S., 2012. A convenient method for simultaneous quantification of multiple phytohormones and metabolites: application in study of rice-bacterium interaction. Plant Methods 8, 2.

Mousavi, S.M., Shabani, L., 2019. Rosmarinic acid accumulation in Melissa officinalis shoot cultures is mediated by ABA. Biol. Plant. 63, 418-424.

Munns, R., Tester, M., 2008. Mechanisms of salinity tolerance. Annu. Rev. Plant Biol. 59, 651-681.

Osakabe, Y., Yamaguchi-Shinozaki, K., Shinozaki, K., Tran, L.S., 2014. ABA control of plant macroelement membrane transport systems in response to water deficit and high salinity. New Phytol. 202, 35-49.

Park, S.C., Kim, Y.H., Kim, S.H., Jeong, Y.J., Kim, C.Y., Lee, J.S., Bae, J.Y., Ahn, M.J., Jeong, J.C., Lee, H.S., Kwak, S.S., 2015. Overexpression of the IbMYB1 gene in an orange-fleshed sweet potato cultivar produces a dual-pigmented transgenic sweet potato with improved antioxidant activity. Physiol. Plant. 153, 525-537.

Per, T.S., Khan, N.A., Reddy, P.S., Masood, A., Hasanuzzaman, M., Khan, M.I.R., Anjum, N.A., 2017. Approaches in modulating proline metabolism in plants for salt and drought stress tolerance: phytohormones, mineral nutrients and transgenics. Plant Physiol. Biochem. 115, 126-140.

Perin, E.C., da Silva Messias, R., Borowski, J.M., Crizel, R.L., Schott, I.B., Carvalho, I.R., Rombaldi, C.V., Galli, V., 2019. ABA-dependent salt and drought stress improve strawberry fruit quality. Food Chem. 271, 516-526.

Ren, C.G., Dai, C.C., 2012. Jasmonic acid is involved in the signaling pathway for fungal endophyte-induced volatile oil accumulation of Atractylodes lancea plantlets. BMC Plant Biol. 12, 128.

Rouphael, Y., Petropoulos, S.A., Cardarelli, M., Colla, G., 2018. Salinity as eustressor for enhancing quality of vegetables. Sci. Hortic. 234, 361-369.

Sah, S.K., Reddy, K.R., Li, J., 2016. Abscisic acid and abiotic stress tolerance in crop plants. Front. Plant Sci. 7, 571.

Shahidi, F., Ambigaipalan, P., 2015. Phenolics and polyphenolics in foods, beverages and spices: antioxidant activity and health effects - a review. J. Funct. Food. 18, $820-897$. 
Shakirova, F.M., Allagulova, C.R., Maslennikova, D.R., Klyuchnikova, E.O., Avalbaev, A. M., Bezrukova, M.V., 2016. Salicylic acid-induced protection against cadmium toxicity in wheat plants. Environ. Exp. Bot. 122, 19-28.

Van Hung, P., 2016. Phenolic compounds of cereals and their antioxidant capacity. Crit. Rev. Food Sci. Nutr. 56, 25-35.

Van Zelm, E., Zhang, Y., Testerink, C., 2020. Salt tolerance mechanism of plants. Annu. Rev. Plant Biol. 71, 403-433.

Wang, X.C., Wu, J., Guan, M.L., Zhao, C.H., Geng, P., Zhao, Q., 2019. Arabidopsis MYB4 plays dual roles in flavonoid biosynthesis. Plant J. 101 (3), 637-652.

Waseem, M., Rong, X., Li, Z., 2019. Dissecting the role of a basic helix-loop-helix transcription factor, SlbHLH22, under salt and drought stresses in transgenic Solanum lycopersicum L. Front. Plant Sci. 10, 734.

Xing, B., Liang, L., Liu, L., Hou, Z., Yang, D., Yan, K., Zhang, X., Liang, Z., 2018. Overexpression of SmbHLH148 induced biosynthesis of tanshinones as well as phenolic acids in Salvia miltiorrhiza hairy roots. Plant Cell Rep. 37, 1681-1692.

Yan, K., Wu, C., Zhang, L., Chen, X., 2015. Contrasting photosynthesis and photoinhibition in tetraploid and its autodiploid honeysuckle (Lonicera japonica Thunb.) under salt stress. Front. Plant Sci. 6, 227.
Yan, K., Cui, M., Zhao, S., Chen, X., Tang, X., 2016a. Salinity stress is beneficial to the accumulation of chlorogenic acids in honeysuckle (Lonicera japonica Thunb.). Front. Plant Sci. 7, 1563.

Yan, K., Xu, H., Zhao, S., Shan, J., Chen, X., 2016b. Saline soil desalination by honeysuckle (Lonicera japonica Thunb.) depends on salt resistance mechanism. Ecol. Eng. 88, 226-231.

Yan, K., Zhao, S., Bian, L., Chen, X., 2017. Saline stress enhanced accumulation of leaf phenolics in honeysuckle (Lonicera japonica Thunb.) without induction of oxidative stress. Plant Physiol. Biochem. 112, 326-334.

Yan, K., Bian, T., He, W., Han, G., Lv, M., Guo, M., Lu, M., 2018. Root abscisic acid contributes to defending photoinibition in Jerusalem artichoke (Helianthus tuberosus L.) under salt stress. Int. J. Mol. Sci. 19, 3934.

Yang, D.F., Ma, P.D., Liang, X., Wei, Z., Liang, Z.S., Liu, Y., Liu, F.H., 2012. PEG and ABA trigger methyl jasmonate accumulation to induce the MEP pathway and increase tanshinone production in Salvia miltiorrhiza hairy roots. Physiol. Plant. 146, 173-183.

Zhu, J.K., 2016. Abiotic stress signaling and responses in plants. Cell 167, 313-324.

Zhu, L., Zhang, Y., Zhang, W., Lu, J., 2016. Effects of exogenous abscisic acid on phenolic characteristics of red Vitis vinifera grapes and wines. Food Sci. Biotechnol. 25, 361-370. 